\title{
TIETZE-TYPE THEOREMS ON MONOTONE INCREASING SETS
}

\author{
JEAN CHAN STANEK
}

\begin{abstract}
The Tietze theorem on convex sets is generalized to monotone increasing sets and strictly monotone increasing sets, which include convex sets as a special case. The main theorem is that a closed connected set in $E_{2}$ is monotone increasing if and only if it is locally monotone increasing. A similar result is proved for strictly monotone increasing sets.
\end{abstract}

1. Introduction and preliminaries. In 1928 Tietze [3] proved that a closed connected set in $E_{n}$ is convex if and only if it is locally convex. Klee [1] generalized this theorem to a topological linear space. We will prove that a closed connected set in $E_{2}$ is monotone increasing if and only if it is locally monotone increasing. A similar result is proved for strictly monotone increasing sets.

Let $E_{2}$ be a two-dimensional Euclidean space with the rectangular coordinate axes. The closure, interior, boundary, and convex hull of a set $S$ in $E_{2}$ are denoted by cl $S$, int $S$, bd $S$, conv $S$, respectively. If $x$ and $y$ are distinct points, then $x y$ denotes the closed line segment joining $x$ and $y$ and intv $x y$ denotes the relative interior of $x y$. For two distinct points $x$ and $y$ in $E_{2}$ not lying on a vertical line, let $m(x, y)=m(L(x, y))$ denote the slope of the line $L(x, y)$ through $x$ and $y$. If $x$ and $y$ are two points in $E_{2}$ not lying on a vertical or horizontal line, the line segment $x y$ determines two closed convex triangles having $x y$ as the hypotenuse and each of the remaining sides parallel to one of the axes. The triangle lying below $x y$ is denoted by $T(x, y)$, called the lower triangle determined by $x$ and $y$. A convex arc $C(x, y)$ joining $x$ and $y$ is called a monotone increasing arc if $m(x, y)>0$ and $C(x, y) \subset T(x, y)$.

DEFINITION 1. A set $S$ in $E_{2}$ is monotone increasing if for each pair of distinct points $x \in S, y \in S$, it is true that

(1) there exists a monotone increasing arc $C(x, y)$ in $S$ joining $x$ and $y$ if $m(x, y)>0$; and

(2) $x y \subset S$ if $x$ and $y$ are on a vertical line or if $m(x, y) \leq 0$.

We note that the arc $C(x, y)$ in (1) of Definition 1 above can contain at most one horizontal line segment since $C(x, y)$ is a convex arc and $C(x, y)$ $\subset T(x, y)$.

DEFINITION 2. A set $S$ in $E_{2}$ is strictly monotone increasing if for each pair of distinct points $x \in S, y \in S$, it is true that

(1) if $m(x, y)>0$, there exists a monotone increasing arc $C(x, y)$ joining $x$

Presented to the Society, April 24, 1971 under the title A Tietze-type theorem on monotone increasing sets; received by the editors July 9, 1974 and, in revised form, November 1, 1974.

AMS (MOS) subject classifications (1970). Primary 52-00, 52A 10.

Key words and phrases. Convex sets, Tietze's theorem, local convexity, monotone increasing sets, strictly monotone increasing sets. 
and $y$ in $S$ where $C(x, y)$ contains no horizontal line segment; and

(2) $x y \subset S$ if $x$ and $y$ are on a vertical line or if $m(x, y) \leq 0$.

DeFinition 3. A set $S$ in $E_{2}$ is locally (strictly) monotone increasing at a point $z \in S$ if there exists a neighborhood $N$ of $z$ such that $N \cap S$ is (strictly) monotone increasing. A set $S$ is locally (strictly) monotone increasing if it is locally (strictly) monotone increasing at each of its points.

We observe that if $S \subset E_{2}$ is an open monotone increasing set, then $S$ is strictly monotone increasing. To see this, let $x$ and $y$ be arbitrary distinct points of $S$. If $x$ and $y$ lie on a vertical line or if $m(x, y) \leq 0$, then $x y \subset S$. Suppose $m(x, y)>0$, and suppose that $C(x, y)$ is a monotone increasing arc joining $x$ and $y$ in $S$ with $y$ lying in the upper half-plane bounded by the horizontal line through $x$. Let $x u$ be the longest horizontal line segment contained in $C(x, y)$. Since $S$ is open, for each $z \in x u$, there exists an open set $N_{z}$ of $z$ such that $N_{z} \subset S$. The compactness of the line segment $x u$ implies the existence of a finite collection of these open sets, say $N_{z_{1}}, \ldots, N_{z_{n}}$, with $x u$ contained in $N_{z_{1}} \cup \cdots \cup N_{z_{n}} \subset S$. Without loss of generality, we assume that $u \in N_{z_{n}}$. Now we choose a point $w$ in $N_{z_{n}} \cap C(u, y)$, where $C(u, y)$ is the subarc of $C(x, y)$ joining $u$ and $y$, such that $w \neq u, w \neq y$ and $x w \subset N_{z_{1}}$ $\cup \cdots \cup N_{z_{n}}$. The set $x w \cup C(w, y)$, where $C(w, y)$ is the subarc of $C(x, y)$ joining $w$ and $y$, is a monotone increasing arc containing no horizontal line segment and joining $x$ and $y$ in $S$. This proves that $S$ is strictly monotone increasing.

To prove our characterization theorems, we apply a result on stripwise subconvex sets [2]. The relevant definitions and theorem are stated below.

Definition 4. A set $S$ in $E_{2}$ is stripwise subconvex if for each pair of distinct points $x$ and $y$ in $S$ there exists a convex $\operatorname{arc} A(x, y)$ in $S$ joining $x$ and $y$ such that

(1) $A(x, y)=x y$ if $x$ and $y$ are on a vertical line; and

(2) if $x$ and $y$ are not on a vertical line, then $A(x, y)$ lies in the closed lower half-plane bounded by the line $L(x, y)$ and $A(x, y)$ lies in the convex hull of the vertical lines through $x$ and $y$ respectively. Such an arc $A(x, y)$ is called a stripwise subconvex arc.

DFFinition 5. A set $S$ in $E_{2}$ is locally stripwise subconvex if for each $z \in S$ there exists a neighborhood $N$ of $z$ such that $N \cap S$ is stripwise subconvex.

THEOREM 1. A closed connected set $S$ in $E_{2}$ is stripwise subconvex if and only if it is locally stripwise subconvex [2].

We note that a monotone increasing set is stripwise subconvex but the converse is not true in general.

If $S \subset E_{2}$ is a closed set and two points $x$ and $y$ in $S$ can be joined by a stripwise subconvex arc in $S$, then there exists a minimal stripwise subconvex arc $C_{0}(x, y)$ joining $x$ and $y$ in $S$ in the sense that if $C(x, y)$ is any other stripwise subconvex arc joining $x$ and $y$ in $S$, it is true that conv $C_{0}(x, y)$ $\subset$ conv $C(x, y)$. The same assertion holds for monotone increasing arcs. To see this, first consider the case when $x y \nsubseteq S$. Let $\{C(x, y)\}$ be the collection of all stripwise subconvex (monotone increasing) arcs joining $x$ and $y$ in $S$, this collection is nonempty by hypothesis. Now set

$$
C_{0}(x, y)=\text { bd }\{\cap \operatorname{conv} C(x, y)\}-\operatorname{intv} x y
$$

where the intersection is taken over all members of the collection. Since the 
set $S$ is closed, we have $C_{0}(x, y) \subset S$. Clearly $C_{0}(x, y)$ is a minimal stripwise subconvex (monotone increasing) arc joining $x$ and $y$ in $S$. In case $x y \subset S$, then $x y$ is the minimal arc required.

The theorem stated below will be useful in the following discussion. A more general form of this theorem can be found in Valentine [4].

THEOREM 2. Let $S$ be a closed convex set in $E_{2}$. Each compact connected portion of the boundary of $S$ which is not contained in a line segment contains an exposed point of $S$. If $S$ is a line segment, it has two exposed points.

\section{The results.}

THEOREM 3. Let $S$ be a closed connected set in $E_{2}$. The set $S$ is monotone increasing if and only if it is locally monotone increasing.

Proof. The necessity is obvious. To prove the sufficiency, let $x$ and $y$ be arbitrary distinct points in $S$. Since $S$ is locally monotone increasing, it is locally stripwise subconvex. By Theorem $1, S$ is stripwise subconvex. If $x$ and $y$ lie on a vertical line, then $x y \subset S$. Otherwise, let $A(x, y)$ be a stripwise subconvex arc joining $x$ and $y$ in $S$. We may assume that $A(x, y)$ is minimal. Now we consider the following cases according to the slope $m(x, y)$.

(a) $m(x, y)>0$. If $A(x, y)=x y$, there is nothing to prove. Suppose that $A(x, y) \neq x y$, and without loss of generality we may assume that $y$ lies in the upper half-plane bounded by the horizontal line through $x$. We will show that $A(x, y)$ is a monotone increasing arc joining $x$ and $y$ in $S$. Let $z$ be an exposed point of conv $A(x, y)$ on $A(x, y)-\{x, y\}$ and let $L$ be a corresponding line of support to conv $A(x, y)$ through $z$ for which $L \cap \operatorname{conv} A(x, y)=\{z\}$. We wish to show that $L$ has positive slope. Suppose on the contrary that $m(L) \leq 0$. Since $S$ is locally monotone increasing, a neighborhood $N$ of $z$ exists such that $N \cap S$ is monotone increasing. We may choose $N$ to be a convex neighborhood such that $x \notin N$ and $y \notin N$. See Figure.

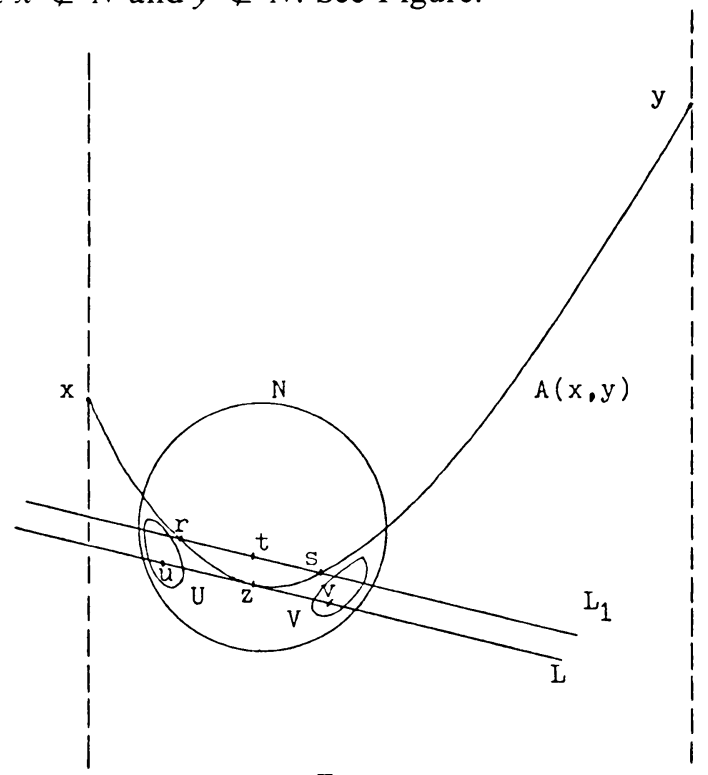

FIGURE 
Since $A(x, y)$ is compact, conv $A(x, y)$ is compact. We can choose points $u$ and $v$ on $L$ with $z \in \operatorname{intv} u v$, and neighborhoods $U$ of $u$ and $V$ of $v$ such that $U \subset N, V \subset N, U \cap \operatorname{conv} A(x, y)=\varnothing$, and $V \cap \operatorname{conv} A(x, y)=\varnothing$. The fact $A(x, y) \neq x y$ implies that int $\operatorname{conv} A(x, y) \neq \varnothing$, thus

$$
\operatorname{cl}(\operatorname{conv} A(x, y))=\operatorname{cl}(\text { int } \operatorname{conv} A(x, y)) .
$$

Hence any neighborhood of $z$ contains an interior point of conv $A(x, y)$. Choose a point $t \in$ int $\operatorname{conv} A(x, y) \cap N$ sufficiently close to $z$ so that there exists a line $L_{1}$ through $t$ parallel to $L$ with $L_{1} \neq L$ such that $L_{1} \cap U$ $\neq \varnothing$ and $L_{1} \cap V \neq \varnothing$. Therefore $L_{1} \cap \operatorname{conv} A(x, y)$ contains no points of $U$ and $V$ and $L_{1} \cap \operatorname{conv} A(x, y) \subset N$. Let $r s$ denote $L_{1} \cap \operatorname{conv} A(x, y)$ where $r$ and $s$ are boundary points of conv $A(x, y)$ and, therefore, points of $A(x, y)$. The line segment $r s$ is nondegenerate since $t \in$ intv $r s$. We have $m(r, s)=m\left(L_{1}\right)$ $=m(L) \leq 0$ since $L_{1}$ is parallel to $L$. Since $N \cap S$ is monotone increasing and $r$ and $s$ lie in $N \cap S$ with $m(r, s) \leq 0$, it follows that $r s \subset S$. Now set $B(x, y)=A(x, r) \cup r s \cup A(s, y)$ where $A(x, r)$ and $A(s, y)$ are the subarcs of $A(x, y)$ joining $x$ to $r$ and $s$ to $y$ respectively. Since $z$ is an exposed point of conv $A(x, y)$ and $z \in A(r, s)$ where $A(r, s)$ is the subarc of $A(x, y)$ joining $r$ and $s$, we have $A(r, s) \neq r s$. The $\operatorname{arc} B(x, y)$ is a stripwise subconvex arc joining $x$ and $y$ in $S$. The existence of $B(x, y)$ contradicts the minimality of $A(x, y)$ since conv $A(x, y)$ is not contained in conv $B(x, y)$. Hence $L$ must have positive slope.

If $A(x, y)$ is not contained in the closed upper half-plane bounded by the horizontal line through $x$, let $w$ be the point of intersection of $A(x, y)$ and the horizontal line through $x$. Thus $A(x, w)-\{x, w\}$ lies in the open lower halfplane bounded by the horizontal line through $x$ where $A(x, w)$ is the subarc of $A(x, y)$ joining $x$ to $w$. Theorem 2 and this fact imply that there exists an exposed point $b$ of $\operatorname{conv} A(x, w)$ on $A(x, w)-\{x, w\}$ with a line of support $K$ to $\operatorname{conv} A(x, w)$ through $b$ such that $K \cap \operatorname{conv} A(x, w)=\{b\}$ and $m(K) \leq 0$. Clearly the point $b$ is an exposed point of conv $A(x, y)$ and the line $K$ is a line of support to conv $A(x, y)$ through $b$ such that $K \cap \operatorname{conv} A(x, y)=\{b\}$. The fact $m(K) \leq 0$ contradicts what we proved above. Hence $A(x, y)$ lies in the closed upper half-plane bounded by the horizontal line through $x$. But $A(x, y)$ is stripwise subconvex, thus $A(x, y)$ must lie in the lower triangle $T(x, y)$ and, hence, $A(x, y)$ is a monotone increasing arc joining $x$ and $y$ in $S$.

(b) $m(x, y)<0$. For $z$ and $L$ described in (a), $L$ has positive slope. From Theorem 2 and the fact $m(x, y)<0$, it follows that $A(x, y)=x y$.

(c) $m(x, y)=0$. As in (a) we conclude that $A(x, y)$ must lie in the closed upper half-plane bounded by the horizontal line through $x$. Consequently $A(x, y)=x y$.

We conclude that $S$ is a monotone increasing set.

THEOREM 4. Let $S$ in $E_{2}$ be a closed connected set. The set $S$ is strictly monotone increasing if and only if it is locally strictly monotone increasing.

Proof. The necessity is obvious. To prove the sufficiency, let $x$ and $y$ be arbitrary distinct points in $S$. By Theorem $3, S$ is monotone increasing. Hence $x y \subset S$ if $x$ and $y$ are on a vertical line or if $m(x, y) \leq 0$. If $m(x, y)>0$, then there exists a monotone increasing arc $C(x, y)$ joining $x$ and $y$ in $S$. Since $S$ is 
closed, we may take $C(x, y)$ to be minimal. We only need to show that $C(x, y)$ contains no horizontal line segment. Assume that $y$ lies in the upper half-plane bounded by the horizontal line through $x$. Suppose $x z$ is the longest horizontal line segment of $C(x, y)$. Since $S$ is locally strictly monotone increasing at the point $z$, there is a neighborhood $N$ of $z$ such that $N \cap S$ is strictly monotone increasing. If $x \neq z$, there exists a point $s \in N \cap$ intv $x z$, and a point $t \in N \cap C(z, y)$ with $t \neq z$ and $t \neq y$ where $C(z, y)$ is the subarc of $C(x, y)$ joining $z$ and $y$. The points $s$ and $t$ lie in $N \cap S$, and $m(s, t)>0$, therefore there exists a monotone increasing arc $D(s, t)$ in $N \cap S$ such that $D(s, t)$ contains no horizontal line segment. Let

$$
D_{0}(s, t)=\operatorname{bd}\{\operatorname{conv} D(s, t) \cap \operatorname{conv} C(s, t)\}-\operatorname{intv} s t
$$

where $C(s, t)$ is the subarc of $C(x, y)$ joining $s$ and $t$. It is obvious that $D_{0}(s, t) \subset S$. Set $C_{0}(x, y)=x s \cup D_{0}(s, t) \cup C(t, y)$, where $C(t, y)$ is the subarc of $C(x, y)$ joining $t$ and $y$. The arc $C_{0}(x, y)$ is clearly convex and it is a monotone increasing arc joining $x$ and $y$ in $S$. But conv $C(x, y)$ is not contained in conv $C_{0}(x, y)$ since $D(s, t)$ and $D_{0}(s, t)$ contain no horizontal line segment. The existence of $C_{0}(x, y)$ contradicts the minimality of $C(x, y)$. Thus $x=z$ and $C(x, y)$ contains no horizontal line segment. Hence $S$ is a strictly monotone increasing set.

\section{BIBLIOGRAPHY}

1. V. L. Klee, Jr., Convex sets in linear spaces, Duke Math. J. 18 (1951), 443-466. MR 13, 354.

2. J. Hutchison, Subconvex sets, Dissertation, University of California, Los Angeles, 1970.

3. H. Tietze, Über Konvexheit im kleinen und im grossen und über gewisse den Punkten einer Menge zugeordnete Dimensionszahlen, Math. Z. 28 (1928), 697-707.

4. F. A. Valentine, Convex sets, McGraw-Hill Ser. in Higher Math., McGraw-Hill, New York, 1964. MR 30 \#03.

Department of Mathematics, California State College, Sonoma, Rohnert Park, CaliFORNIA 94928 